\title{
Liquid-based hybridization assay with real-time detection in miniaturized array platforms
}

\author{
Anna Guerasimova ${ }^{a}$, Lajos Nyarsik ${ }^{b}$, Jian-Ping Liu ${ }^{a, c}$, Regine Schwartz ${ }^{b}$, \\ Matthias Lange ${ }^{\mathrm{b}}$, Hans Lehrach ${ }^{\mathrm{b}}$, Michal Janitz ${ }^{\mathrm{a}, *}$ \\ ${ }^{a}$ Max Planck Institute for Molecular Genetics, Fabeckstrasse 60-62, 14195 Berlin, Germany \\ ${ }^{\mathrm{b}}$ Max Planck Institute for Molecular Genetics, Ihnestrasse 73, 14195 Berlin, Germany \\ ${ }^{\mathrm{c}}$ FU Berlin, Department of Biology, Chemistry and Pharmacy, Takustr. 3, 14195 Berlin, Germany
}

Received 7 June 2005; received in revised form 26 September 2005; accepted 26 September 2005

\begin{abstract}
An assay for the fluorescent detection of short oligonucleotide probe hybridization in miniaturized high-density array platforms is presented. It combines hybridization in solution with real-time fluorescent detection, which involves measurement of fluorescence increase by means of an induced fluorescence resonance energy transfer. The feasibility of this approach using DNA or RNA as a target, and short DNA- as well as LNA (locked nucleic acid)-modified oligonucleotides as probes is shown. The presented approach could potentially contribute to a significant increase in the throughput of large-scale genomic applications, such as oligofingerprinting and genotyping, and also reduce material consumption.

(C) 2005 Elsevier B.V. All rights reserved.
\end{abstract}

Keywords: Hybridization; iFRET; Locked nucleic acids; Oligonucleotide fingerprinting

Nucleic acid hybridization with oligonucleotides is a fundamental method in DNA analysis and is applied to a variety of applications: from the quantitative comparison of diversity within a complex sample (such as in expression profiling), to locating the fine differences between multiple identical samples (such as in genotyping and re-sequencing). The predominant format of these assays is the high-density microarray of pre-fabricated immobilized oligonucleotide probes by which complex (Wong et al., 2004) or single (Pe'er et al., 2003) targets are interrogated through hybridization. Detection of hybridization on arrays is based on either direct or secondary (with additional conjugates coupled to fluorescent dyes) fluorescence. Most of the conventional fluorescent labels, however, are characterized by low sensitivity, especially on membranes or glass arrays due to poor signal to noise ratios.

In the present study, a method for in-solution analysis of DNA libraries by means of hybridization with short (6-8 nucleotides long) fluorescent oligonucleotide probes with realtime optical detection by induced fluorescence resonance transfer (iFRET) (Howell et al., 2002) has been established.

\footnotetext{
* Corresponding author. Tel.: +49 308413 1463; fax: +49 3084131462. E-mail address: janitz@molgen.mpg.de (M. Janitz).
}

Only hybridized probe is detected as its fluorescent signal is generated through the excitation of the intercalating dye when a hybridization event takes place. Thus, elimination of unreacted excess probe is not necessary. The original procedure (Howell et al., 2002) for the analysis of long DNA templates has been also modified, so that immobilization of DNA and strand separation is avoided. Therefore, the assay can be performed entirely in solution. It is essentially independent from the surface binding capacity or DNA immobilization chemistry, requiring only accurate dispensing of both probe and target.

Maximizing the advantages of the superior hybridization properties of locked nucleic acids (LNAs) (Nielsen et al., 1999), probes that were modified with LNA bases for iFRET detection, were evaluated. Due to the high affinity of these probes, very short probes can potentially be used for hybridization analysis (Simeonov and Nikiforov, 2002). This can be useful, in particular, for single nucleotide polymorphism (SNP) typing schemes or when multiple probes with the size of universal probe sets (e.g. in sequencing by hybridization method (Pe'er et al., 2003)) are considered.

Yet, another aspect of our work was to minimize the cost of screening, which has been addressed primarily by reducing the volumes of samples. Technology development leads to conversion from a limited number of reactions in a greater volume to 
extremely large numbers of assays performed in nanolitre and picolitre volumes. This is an enormous advantage for probe/ sample analysis that involves a high-throughput, such as oligonucleotide fingerprinting (ONF) (Meier-Ewert et al., 1998; Herwig et al., 2000, 2002), in which a minimum of 100,000 clones have to be probed individually with at least 100 oligonucleotides, which means that $10^{7}$ independent assays have to be run.

The traditional ONF method, which is based on radioactive oligonucleotide hybridizations using DNA clones that have been immobilized on nylon membrane filters, is limited by the vast experimental costs and time consumption. The conceived nano-well format, combined with a one-step protocol of fluorescent hybridization detected by a sensitive chargecoupled device (CCD) camera, should reduce the experimental load by a factor of $10^{3}$ (in comparison with a microplate approach), reducing the load from $10 \mu \mathrm{l}$ to $10 \mathrm{nl}$. In this work, the method of hybridization with short oligonucleotide probes and iFRET detection is demonstrated, which can be used in micro-droplets and nano-well-array formats and can be applied to DNA clone characterization.

DNA and DNA-LNA oligoprobes were synthesized by MWG-Biotech (Germany) or TIB MolBiol (Germany). The sequences of probes and DNA targets used in this work are shown in Table 1. All probes were $5^{\prime}$-end labelled using Cy5, ROX or Bodipy fluorescent dyes. The templates used in the present study were synthetic DNAs of 24-40 nucleotides in length or DNA clones of 1-2 kb in length, with a previously published sequence (Bauer et al., 2004) being complementary to the respective oligo-sequences. DNA clones were amplified and purified using a PCR Purification Kit (Qiagen, Germany) and converted into RNA using a Message $\mathrm{Amp}^{\mathrm{TM}}$ Kit (Ambion). Subsequent fragmentation was carried out by incubation for $20 \mathrm{~min}$ at $94{ }^{\circ} \mathrm{C}$ in a fragmentation buffer (5× : $200 \mathrm{mM}$ Tris-acetate, $\mathrm{pH} 8.1,500 \mathrm{mM}$ KOAc, $150 \mathrm{mM}$ $\mathrm{MgOAc})$. The fragmentation products were analysed using an Agilent 2100 Bioanalyser (Agilent Technologies, USA) and quantified using a Bio-Photometer (Eppendorf, Germany).

Hybridization experiments were carried out in a hybridization buffer (50 mM NaCl, 0.1\% Tween-20, 50 mM Tris-HCl, pH 7.5) containing Sybr Green I at 1:1000 dilution. Real-time evaluation of hybridizations was performed in a volume of $100 \mu \mathrm{l}$ hybridization buffer in quartz cuvettes (Qs $10 \mathrm{~mm}$; Hellma, Germany). The hybridizations were detected by thermocontrollable spectrophotometer (Avantes Fiberoptic, The Netherlands) and processed using Avasoft 5.1 software. In-droplet hybridization format was performed in a volume of $2 \mu \mathrm{l}$ hybridization buffer. In this case, both probe and target solutions at $1 \mu \mathrm{l}$ volumes were pipetted or dispensed onto an air-dried glass slide surface that had been pre-washed with $100 \%$ ethanol (Menzel, Germany). The slides were then covered with a second glass slide, spaced with an adhesive tape providing a distance of $255 \mu \mathrm{m}$ between the two slides to prevent evaporation. The slides were placed onto a thermal cycler (Eppendorf) that was set to a heating increment of $3{ }^{\circ} \mathrm{C} / \mathrm{min}$. The final concentrations in the hybridization mixture were: $0.5-1.5 \mu \mathrm{M}$ for probes and $0.15-$ $0.5 \mu \mathrm{M}$ for the targets (synthetic DNAs or RNAs), for both the micro-drop and nano-well formats.
Table 1

Oligonucleotide probes and synthetic DNA targets used in the study

\begin{tabular}{|c|c|}
\hline \multicolumn{2}{|r|}{ Oligonucleotide probes } \\
\hline OP-1 & Cy5-TTGCCAA \\
\hline OP-2 & $\mathrm{Cy} 5-\mathrm{TG}^{\#} \mathrm{CCA}^{\#} \mathrm{~A}$ \\
\hline OP-3 & Cy5-TCAGAAG \\
\hline OP-4 & $\mathrm{Cy} 5-\mathrm{CA}^{\#} \mathrm{GAA} \mathrm{A}^{\#} \mathrm{G}$ \\
\hline OP-5 & Cy5-TCCTCCTG \\
\hline OP-6 & Су5-ТССТCCТ \\
\hline OP-7 & Су5-ТССТСC \\
\hline OP-8 & Cy5-T" $\mathrm{CCTCC}$ \\
\hline OP-9 & Cy5-ATGAGGA \\
\hline OP-10 & Cy5-CCTGCCC \\
\hline OP-11 & Cy5-GCTCGTT \\
\hline OP-12 & Bodipy-AGCCGATTG \\
\hline OP-13 & ROX-CTCCAAG \\
\hline OP-14 & Cy5-CTGAAGC \\
\hline OP-15 & Cy5-C ${ }^{\#}$ TGAAG $^{\#} \mathrm{G}$ \\
\hline OP-16 & Cy5-TGCTGGT \\
\hline OP-17 & Су5-СТССТСС \\
\hline OP-18 & Cy5-GCTCCTG \\
\hline \multicolumn{2}{|r|}{ Targets } \\
\hline DNA-1 & 5'-TTTGCAGGAAGAGTC CCAATCGGCTTT \\
\hline DNA-2 & 5'-TTTGCAGGAGGAGTC CCAATCGGCTTT \\
\hline DNA-3 & 5'-TAGTTCTTGGAGAAGG \\
\hline DNA-4 & 5'-TCCTCATTCCAGGAACACAGCAGC \\
\hline DNA-5 & 5' TTCACCAGCAATGCTCCTCATTGC \\
\hline DNA-6 & 5'-TTTGGCTGCGGCACAGTGACAGGAGCA \\
\hline DNA-7 & 5'-CCCAAATAACATTCACCAGCATTGGAGGAGGTT \\
\hline DNA-8 & 5'-TGCTTTGTCACACCAGCCACTCACACTGGGGAAG \\
\hline DNA-9 & 5'-CACCAGCATCCTCATTGGGAGAGACAGAGAAA \\
\hline
\end{tabular}

Modifications with LNA bases are indicated with \#.

For nano-dispensing, the TopSpot/E Micro-Arrayer module (IMTEK, Germany) was used. Oligonculeotides and DNA targets were dispensed into wells at volumes of $4 \mathrm{nl}$ in two directions on the nano-well plate, which was pre-filled with $30 \mathrm{nl}$ of buffer containing Sybr Green I dye. Finally, the plate was covered with mineral oil (Sigma, Germany) to prevent 
evaporation. Final concentrations of the reagents were the same as in the micro-drop experiments.

For imaging, a CCD camera of type CH350 (Photometrix Ltd., UK) that had been cooled to $-40{ }^{\circ} \mathrm{C}$ was used, which was integrated with a thermal cycler (Eppendorf) and operated by controlling software that was developed in-house. The camera had a chip size of $512 \times 512$ pixels and an object field of $400 \mathrm{~mm}^{2}$ could be detected. The excitation light was generated in a halogen light source with an interference filter. The emission filter was placed directly to the front side of the camera objective. For excitation, 480 and $620 \mathrm{~nm}$ filters were used and for emission, 540 and $670 \mathrm{~nm}$ filters (Andover Corporation, USA). The images were evaluated using an UTHSCASA Image Tool v3.00 (University of Texas Health Science Center, TX, USA).

The iFRET was originally applied for the detection of hybridization of 15-mer probes with single-stranded DNA targets of comparable lengths, starting from 50 bases (Howell et al., 2002). Using this method, fluorescence of the dye attached to the probe is detected through excitation of an intercalating dye, the fluorescence of which is immensely enhanced following binding to the DNA duplex. Howell et al. used Sybr Green I (Molecular Probes, OR, USA) as a FRET donor, together with ROX (6-rhodamine) or Bodipy TMR as acceptor dyes. Here, the range of possible acceptor dyes was extended by using Cy5. Despite its apparent insufficient overlap of spectra with Sybr Green I emission (Cy5 absorption maximum is at $646 \mathrm{~nm}$, and Sybr Green I emission maximum is at $518 \mathrm{~nm}$ ), our results show that it could be used as an acceptor in a FRET pair with Sybr Green I. This is probably due to the fact that the Cy5 dye, when attached to DNA, acquires additional shorter wavelength absorption peaks compared with the spectrum of the free dye (Molecular Probes Handbook, 2005). Having tested various combinations, it has been realized that, for our approach, Cy5 is the best acceptor dye for Sybr Green I. It provided a high iFRET signal, as well as a good spectral separation of the signal from the excitation dye peak, so it was possible to monitor both signals with appropriate filters. Hence, the Sybr Green I signal can be used for quantification and normalization purposes, thereby providing an additional control for the hybridization process. In principle, appropriate filtering should allow detection of more than one acceptor dye, which allows multiplexing of hybridization probes. This possibility was examined for three fluorescent dyes as labels for oligonucleotides: Bodipy, ROX and Cy5. Emission filters with a maximum at $560 \mathrm{~nm}$ for Bodipy TMR fluorescence, at $630 \mathrm{~nm}$ for the ROX filter and at $670 \mathrm{~nm}$ for $\mathrm{Cy} 5$ were used. Fig. 1 shows detection of hybridization using three probes simultaneously. Synthetic DNA oligonucleotides that are specific for a distinct oligonucleotide probe were probed with either an equimolar mixture of oligonucleotides (Fig. 1) or separately with each probe (not shown). As the emission of Bodipy dye is close to the emission of Sybr Green I, it is often difficult to distinguish between these two signals, making signal measurements from Bodipylabelled probes impossible. It has been concluded that most reliable data were obtained using a combination of ROXand Cy5-labelled probes (not shown). Further steps to increase

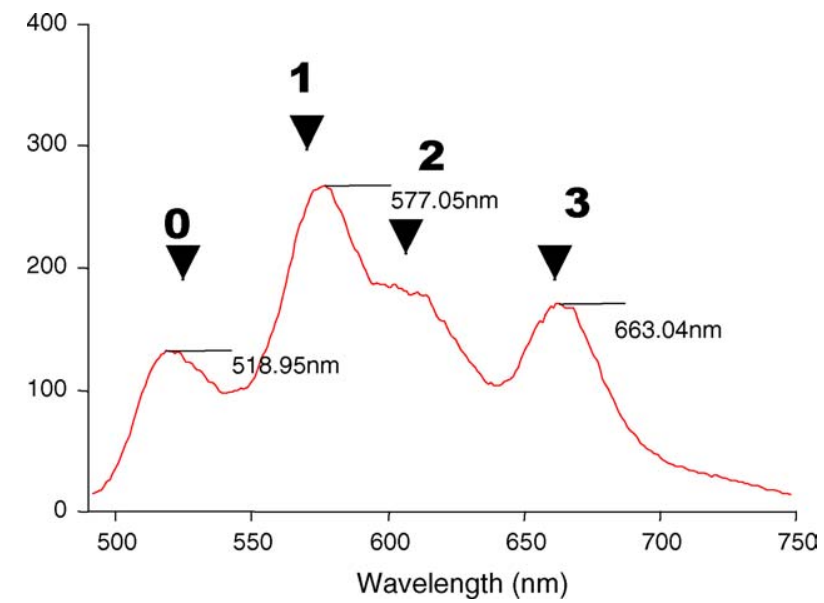

Fig. 1. Hybridization of three pooled 7-mer oligonucleotides labelled with Cy5, Bodipy or ROX fluorescent dyes to two synthetic DNA targets (DNA-2 and DNA-3, Table 1). Oligonucleotide probes were used at equal concentrations, and in 10-fold excess compared with DNA targets. Hybridization was performed in a $100 \mu \mathrm{l}$ volume in a cuvette and detected by spectrophotometer. The observed peaks are: $0(518.95 \mathrm{~nm})$ from Sybr Green I; $1(577.05 \mathrm{~nm})$ from Bodipy-oligo; 2 (not identified, as it overlaps with the Bodipy-oligo emission) ROX-oligo; and $3(663.04 \mathrm{~nm})$ from Cy5 oligo hybridization. The $Y$-axis represents signal intensity units.

\section{5'- TTTGCAGGAAGAGTCCCAATCGGCTTT mismatch (DNA1)}

5'- TTTGCAGGAGGAGTCCCAATCGGCTTT perfect (DNA2)

5'- TCCTCATTCCAGGAACACAGCAGC unrelated (DNA4)

DNA-8-mer Cy5-TCCTCCTG (OP-5)

DNA-7-mer Cy5-TCCTCCT (OP-6)

DNA-6-mer Cy5-TCCTCC (OP-7)

(A) LNA-DNA 6-mer Cy5-T" CCTCC (OP-8)

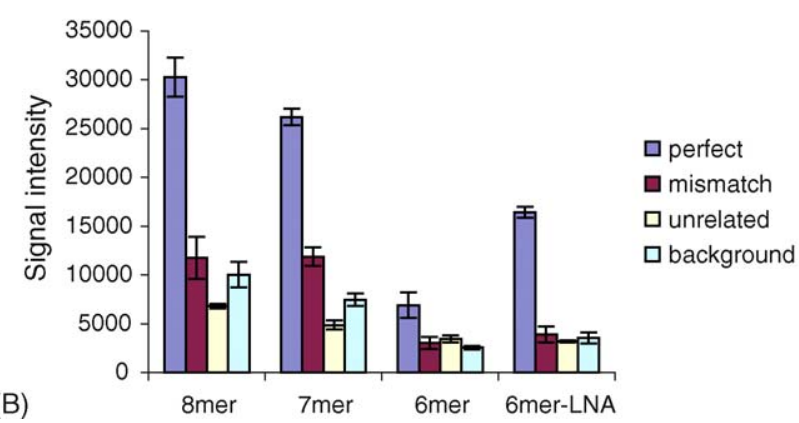

Fig. 2. Assessment of minimal oligonucleotide probe length suitable for iFRET detection. (A) Oligonucleotides and DNA targets (abbreviations indicated in brackets) used for this experiment. Probes of different length but with the same 6-mer core were used for hybridization with three DNA targets: perfectly matched, with a single mismatch (indicated in bold) and unrelated (no match). Sequence fragment complementary to the oligonucleotide probes was underlined. (B) Hybridization results for probes with different length. DNA hybridization was performed in micro-droplets. For each probe, a background signal from the probe and Sybr Green I in solution without DNA was also measured and subtracted from the signal of the probe-DNA mixture. Data are presented from three independent experiments. 
the throughput of the system would be to develop a multi-dye system that could allow simultaneous detection of many intercalation dye/fluorescent dye energy-transfer pairs that are spectrally well-resolved (Shchepinov and Korshun, 2001) or to use the Qbead system (Xu et al., 2003).

As guidelines for the selection of the probe length and base content, information content criteria was used (Herwig et al., 2000), with the requirement that the oligonucleotide GC content should be at least $40 \%$ and that good performance in hybridization experiments in previous studies has been shown (Bauer et al., 2002). Reduced length of the probe should diminish the total number of probes that are necessary to characterize particular DNA fragments in sequencing by hybridizationrelated applications, for example in ONF (Meier-Ewert et al., 1998). Ideally, 6- or 5-mer probe sets should be used. Short oligonucleotides, however, are less specific and less accurate, which results in high signal noise and impeded data analysis. Even if hybridization of DNA oligonucleotides as short as 6-mer (Drmanac et al., 1990) or even 5-mer (Pe'er et al., 2003) were described, probe length is usually limited to 8-10 nucleotides due to the low stability of very short duplexes. Another concern referred to minimal probe length, which would permit detection with iFRET. The presence of Sybr Green I was described to have

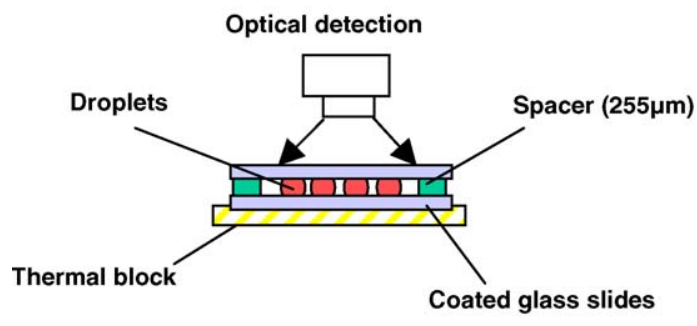

(A) a stabilizing effect on DNA duplexes, resulting in an effective increase of its melting temperature (Prince et al., 2001), which should be advantageous for use of very short oligonucleotides. In fact, it was possible to obtain reliable hybridization of oligonucleotide probes as short as 6- and 7-mer in length (Fig. 2). Hybridization results with 8-, 7- and 6-mer probes sharing the same 6-mer sequence showed a clear correlation of signal decrease with the probe length.

The use of DNA analogues (Guerasimova et al., 2001) or modified DNA bases often helps to enhance hybridization properties of oligonucleotides. For example, the different nature of the LNA that stack in the heteroduplex results in their enhanced performance in terms of stability in a duplex, even for very short probes (Simeonov and Nikiforov, 2002). The sensitivity of LNA in mismatch discrimination makes them particularly attractive substitutes for conventional DNA probes in hybridization-based analysis. LNA-DNA chimerical oligonucleotides with a total length of 7-5 bases have been examined. Alternating DNA and LNA bases within the oligonucleotide sequence was shown to improve its performance (Valoczi et al., 2004). It was taken into consideration when designing our probes, in that LNA bases were always spaced with DNA bases. A typical signal comparison between
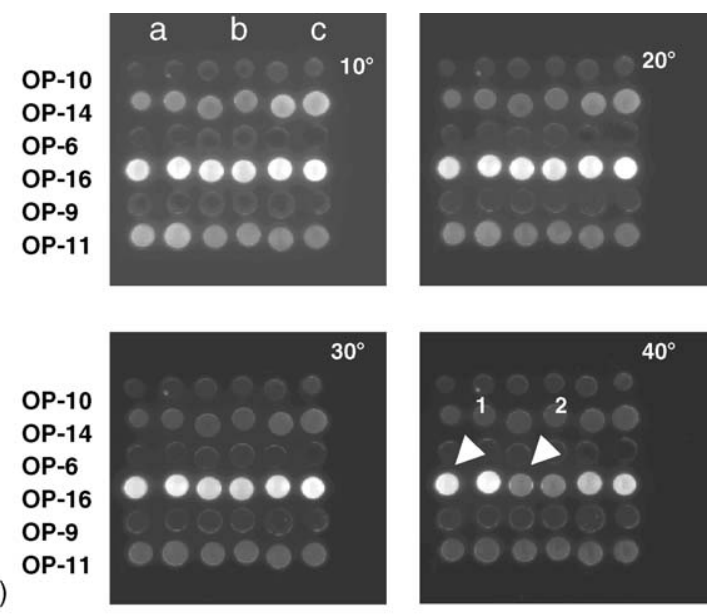

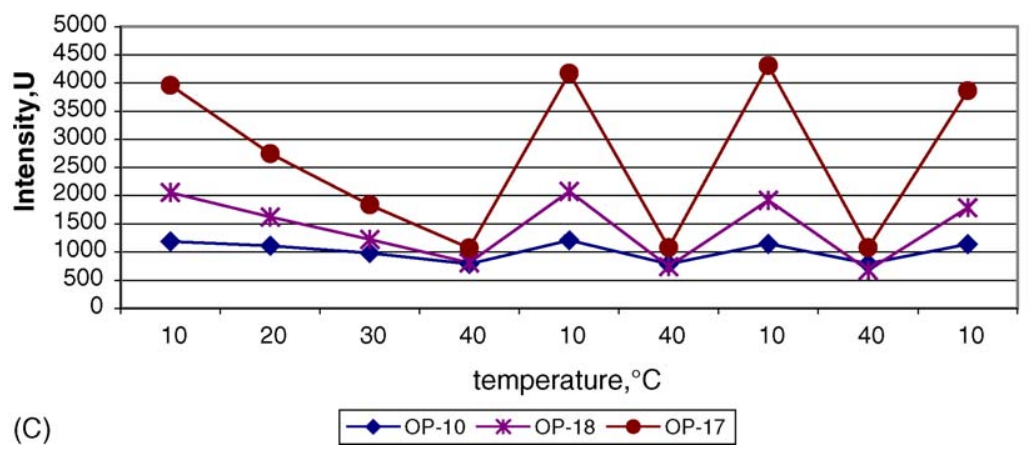

Fig. 3. (A) Scheme of the 'sandwich' in the droplet experiment set-up. (B) Monitoring of the hybridization experiment following heating. Six probes (in $X$-direction), 7-mer Cy5-labelled oligonucleotides (OP-6, OP-9, OP-10, OP-11, OP-14 and OP-16; Table 1) were hybridized with three DNA targets (in $Y$-direction a (DNA-7); b (DNA-8); and c (DNA-9); Table 1) in duplicates. Image acquisition was performed at the different temperatures as shown $\left(10-40{ }^{\circ} \mathrm{C}\right)$. For most of the probes, the result of hybridization should be negative (no matching sites), but for the probe OP-16, targets a and c should be positive, whereas DNA b is a single mismatch. Different signals from perfect and mismatched duplexes ( 1 and 2, as indicated by the arrows) are better discriminated at higher temperatures (e.g. $\left.40{ }^{\circ} \mathrm{C}\right)$. (C) Fluorescence-monitored melting profiles of duplexes formed by the same fragmented RNA template with three different oligonucleotides. Individual hybridizations with three probes were performed in droplets. The starting temperature of hybridization was $10^{\circ} \mathrm{C}$ and then it underwent cyclic changes to $40{ }^{\circ} \mathrm{C}\left(\mathrm{rate}\right.$ of $\left.1.5{ }^{\circ} \mathrm{C} / \mathrm{min}\right)$ and back to $10{ }^{\circ} \mathrm{C}$. The data were collected for 10, 20, 30 and $40^{\circ} \mathrm{C}$ temperature points. The highest signal intensity ( $Y$-axis) was obtained with the fully matched probe OP-17. 


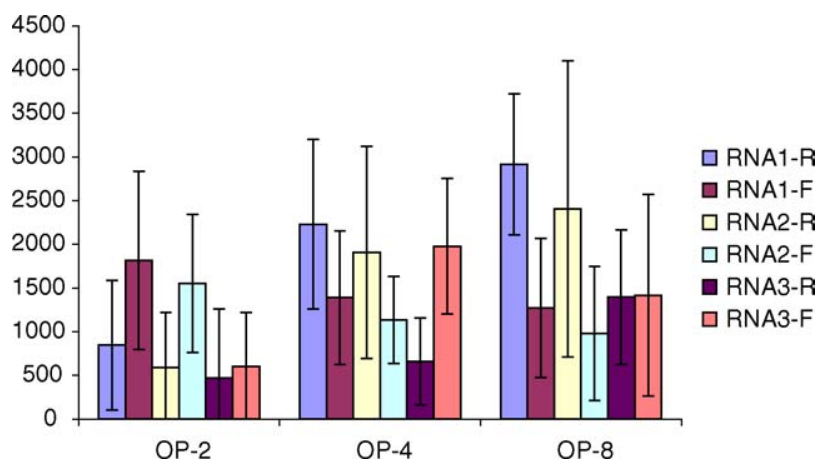

Fig. 4. Sequence-dependent hybridization profiles obtained for six RNAs with three LNA-modified oligonucleotides (6-mers: OP-2, OP-4 and OP-8; Table 1). The RNA1-F and RNA1-R were produced from the same original DNA clone via transcription from the $5^{\prime}$ - or $3^{\prime}$-end, respectively. Hence, six different RNAs originating from three DNA clones were analysed. Hybridizations were performed in droplets, as described in the text. The background fluorescence of the probe and Sybr Green I only was subtracted. Average values of intensities are shown with standard deviations.

LNA-containing and normal DNA oligonucleotides is shown in Fig. 2. It was observed that even 6-mer oligonucleotides, modified only with one LNA base, delivered much stronger signal than non-modified DNA oligonucleotide of the same sequence and was comparable to that of a 7-mer DNA probe. Pentamers (pure DNA or LNA-modified) gave rather weak signals (not shown), so in future, it will be continued to predominantly use 7 - or 6-mer probes.

The standard hybridization assay using Cy5-labelled probes was performed by tracking the changes of fluorescence at both emission lines-540 and $670 \mathrm{~nm}$. Two optical filters were used and the images were captured at different stages of applied temperature ramp $\left(10,20,30\right.$ and $40{ }^{\circ} \mathrm{C} ; 3 \mathrm{~min}$ at each point). Routinely, hybridizations in a $2-\mu 1$ micro-droplet volume placed on a glass slide were performed, with each sample being set-up in duplicate (set-up is shown in Fig. 3A). The monitoring of oligoprobe hybridization with different DNA targets in droplets is shown in Fig. 3B. Melting profiles obtained via repeated heating and cooling of the RNA:DNA duplexes are shown in Fig. 3C for three different oligoprobes.

To evaluate the applicability of our method for OFP analysis, a pilot study on a limited number of selected genomic DNA clones was performed. Fragmented RNA templates (RNA 1-3; Fig. 4) have been generated from genomic DNA clones of known sequences, which were described previously in detail by Bauer et al. (2004). According to the sequence similarity, the initial clones were grouped into clusters. In this way, RNA2 and RNA3 (originating from DNA clones of 1001 and 1344 nucleotides in length) belong to the same cluster, whereas the sequence of RNA1 (1259 nucleotides) was not related to RNA2 and 3. RNAs were generated from three DNA clones by in vitro transcription from both ends of each respective clone, resulting in six RNA templates. Thus, additional control of hybridization specificity was provided. Through converting the template to RNA, the following was achieved: (i) using amplification, the amount of template was increased; (ii) the template was singlestranded, facilitating hybridization with short oligoprobes; (iii)

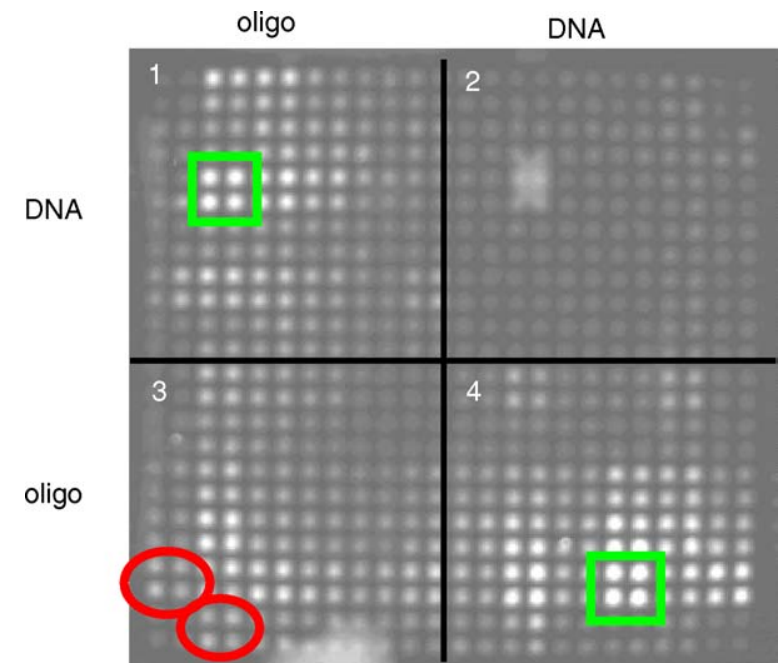

Fig. 5. An image of hybridization in a $24 \times 24$ nano-well plate. DNA targets and oligonucleotides were dispensed with 96 channels using a TopSpot dispensing head. In fields 1 and 4, both DNA and oligoprobes (whole hybridization mixture) are dispensed, whereas in fields 2 and 3, only oligonucleotides or DNAs, respectively, are dispensed (together with Sybr Green I). Hybridization conditions were the same as in the droplet experiments. For normalization of positive hybridization signals (fields 1 and 4; signal locations indicated by a green square), the background signal was substracted from the samples containing no target DNA (sample position marked with red circles). The first two columns in both fields 1 and 2 were buffer only.

RNA could be chemically fragmented, which usually improves the kinetics of hybridization with short oligoprobes.

These RNA samples were hybridized with a small set of oligonucleotides. Graphical representation of different RNA hybridization profiles with three probes is shown in Fig. 4. Clearly, it is possible to distinguish between templates originating from the same (such as RNA2-F and RNA3-F) or different (such as RNA1-F and RNA1-R) sequences.

The micro-droplet format enabled us to reduce the reaction volume below $100 \mathrm{nl}$ using rapid dispensing techniques. For high-density arrays, the nano-well format offers a suitable platform in nanolitre volume range. The method has been verified for nano-well plates using $500 \mu \mathrm{m}$ pitch (400 nano-wells $/ \mathrm{cm}^{2}$ ) and $36 \mathrm{nl}$ total volume per well. Fig. 5 presents an image acquired by fluorescence detection from hybridization screening of five templates with five oligonucleotides (each sample in duplicates) dispensed in two directions into a nano-well plate.

As well as the potential application in ONF technique, the assay described here provides an option for multiplexing and micro-volume processing to downscale the costs. It could potentially be used to create ultra-high-throughput screening, which is necessary for applications such as SNP detection or resequencing (Shendure et al., 2004). In conclusion, the proposed method can be used as a robust platform for in-solution miniaturized sequence-specific clone characterization that is suitable for automation in high-throughput applications.

\section{Acknowledgements}

The study has been supported by the European Commission (integrated project MolTools) and the BMBF (01KW9913). 
The authors are particularly grateful to O. Landt (TIB MolBiol) for help with spectrophotometer measurements and discussions at the initial stage of this project.

\section{References}

Bauer, O., Janitz, M., Guerasimova, A., Herwig, R., Lehrach, H., Radelof, U., 2002. In: Lorkowski, S., Cullen, P. (Eds.), Analysing Gene Expression: A Handbook of Methods. Wiley-VCH, Weinheim.

Bauer, O., Guerasimova, A., Sauer, S., Thamm, S., Steinfath, M., Herwig, R., Janitz, M., Lehrach, H., Radelof, U., 2004. Rapid Commun. Mass Spectrom. $18,1821-1829$.

Drmanac, R., Strezoska, Z., Labat, I., Drmanac, S., Crkvenjakov, R., 1990. DNA Cell Biol. 9, 527-534.

Guerasimova, A., Nyarsik, L., Girnus, I., Steinfath, M., Wruck, W., Griffiths, H., Herwig, R., Wierling, C., O'Brien, J., Eickhoff, H., Lehrach, H., Radelof, U., 2001. Biotechniques 31, 490-495.

Herwig, R., Schmitt, A.O., Steinfath, M., O’Brien, J., Seidel, H., Meier-Ewert, S., Lehrach, H., Radelof, U., 2000. Bioinformatics 16, 890-898.

Herwig, R., Schulz, B., Weisshaar, B., Hennig, S., Steinfath, M., Drungowski, M., Stahl, D., Wruck, W., Menze, A., O'Brien, J., Lehrach, H., Radelof, U., 2002. Plant J. 32, 845-857.

Howell, W.M., Jobs, M., Brookes, A.J., 2002. Genome Res. 12, 1401-1407.
Meier-Ewert, S., Lange, J., Gerst, H., Herwig, R., Schmitt, A., Freund, J., Elge, T., Mott, R., Herrmann, B., Lehrach, H., 1998. Nucleic Acids Res. 26, 22162223.

Molecular Probes Handbook, 2005. http://probes.invitrogen.com/handbook/ boxes/1747.html.

Nielsen, C.B., Singh, S.K., Wengel, J., Jacobsen, J.P., 1999. J. Biomol. Struct. Dyn. 7, 175-191.

Pe'er, I., Arbili, N., Liu, Y., Enck, C., Gelfand, C.A., Shamir, R., 2003. Nucleic Acids Res. 31, 5667-5675.

Prince, J.A., Feuk, L., Howell, W.M., Jobs, M., Emahazion, T., Blennow, K., Brookes, A.J., 2001. Genome Res. 11, 152-162.

Shchepinov, M.S., Korshun, V.A., 2001. Nucleosides Nucleotides Nucleic Acids 20, 369-374.

Shendure, J., Mitra, R.D., Varma, C., Church, G.M., 2004. Nat. Rev. Genet. 5, 335-344.

Simeonov, A., Nikiforov, T.T., 2002. Nucleic Acids Res. 30, e91.

Valoczi, A., Hornyik, C., Varga, N., Burgyan, J., Kauppinen, S., Havelda, Z., 2004. Nucleic Acids Res. 32, e175.

Wong, K.K., Tsang, Y.T., Shen, J., Cheng, R.S., Chang, Y.M., Man, T.K., Lau, C.C., 2004. Nucleic Acids Res. 32, e69.

Xu, H., Sha, M.Y., Wong, E.Y., Uphoff, J., Xu, Y., Treadway, J.A., Truong, A., O’Brien, E., Asquith, S., Stubbins, M., Spurr, N.K., Lai, E.H., Mahoney, W., 2003. Nucleic Acids Res. 31, e43. 